\title{
Variability of Essential and Nonessential Fatty Acids of Irish Rapeseed Oils as an Indicator of Nutritional Quality
}

\author{
Rebecca Coughlan $\mathbb{D}^{1,2}$ Siobhan Moane $\mathbb{D}^{2,3}$ and Tracey Larkin $\mathbb{D}^{1,2,3}$ \\ ${ }^{1}$ FooDs@LIT, Technological University of the Shannon, Midlands Midwest, Ireland \\ ${ }^{2}$ Shannon Applied Biotechnology Centre, Technological University of the Shannon, Midlands Midwest, Ireland \\ ${ }^{3}$ Department of Applied Science, Technological University of the Shannon, Midlands Midwest, Ireland
}

Correspondence should be addressed to Rebecca Coughlan; rebecca.coughlan@lit.ie

Received 26 August 2021; Revised 12 November 2021; Accepted 6 December 2021; Published 12 January 2022

Academic Editor: Mohamad Djaeni

Copyright (c) 2022 Rebecca Coughlan et al. This is an open access article distributed under the Creative Commons Attribution License, which permits unrestricted use, distribution, and reproduction in any medium, provided the original work is properly cited.

\begin{abstract}
The low saturated fatty acid content of rapeseed oil has resulted in it being classed as one of the most health-benefiting culinary oils. This study determines whether Irish rapeseed oils contain identical fatty acid profiles or whether distinct profiles exist between producers and producers' successive oil batches. The fatty acid content of Irish rapeseed oils was determined in terms of the desirable MUFA and PUFA and saturated content of these oils. The fatty acid composition demonstrated significant differences in individual unsaturated fatty acid content, while total saturation had insignificant differences. Saturated fatty acid content ranged from 6.10 to $15.8 \%$, while unsaturated fatty acids ranged from 84.20 to $90.10 \%$. Moreover, individual fatty acid content exhibited significant differences $(p<0.05)$. Oleic acid $(\mathrm{C} 18: 1)$, linoleic acid $(\mathrm{C} 18: 2)$, and stearic acid $(\mathrm{C} 18: 0)$ contents were considered significantly different from other fatty acids detected. The third successive batch from each producer exhibited lower oleic acid content, and the third batch contained higher linoleic acid content, at the same time maintaining a desirable unsaturated fatty acid composition. Studies suggest that differences in the fatty acid composition may be due to cultivation practices such as climate, soil composition, sowing and harvesting, processing techniques, and oxidation reactions.
\end{abstract}

\section{Introduction}

Lipids are a fundamental source of nutrition directly influencing health [1]. Culinary oils composed of saturated fatty acids (SFA) give rise to low-density lipoproteins (LDLs), elevating cholesterol levels and leading to coronary diseases. In comparison, the unsaturated fatty acid (USFA) portion has lower cholesterol levels and is essential [1]. Many epidemiological studies emphasise the nutritional benefits of USFA, with a strong focus on the necessity of polyunsaturated fatty acids (PUFA) as they are not synthesised in the body and must be obtained directly from food [2]. Culinary oils that contain higher levels of PUFA can be considered to have a higher nutritional value. In Ireland, coronary heart disease (CHD) is one of the highest causes of death due to inadequate diet; therefore, demands for health benefiting oils such as rapeseed oil have increased [3].
Rapeseed oil is the second leading culinary oil produced globally as it contains a desirable fatty acid composition of predominantly unsaturated fatty acids and a healthy bioactive compound content [4]. Studies investigating the fatty acid composition of rapeseed oils suggest the total USFA portion can range from $92.2 \%$ to $93.7 \%$, with a higher PUFA content than other culinary oils [5-7]. Oleic acid (C18:1) is reported in numerous publications for its beneficial activity on LDL cholesterol [8]. The fatty acid profile of rapeseed oils has been shown to contain higher oleic acid (C18:1) and linoleic acid (C18:2) levels than other fatty acids, indicating that rapeseed oil is of high nutritional value [9]. Studies have proven that fatty acids are an essential part of the daily dietary requirement due to their significant biological function [2]. Many epidemiology studies have focused on the nutritional benefits of unsaturated fatty acids, with a strong focus on PUFAs, such as linoleic acid and linolenic acid, as they must be obtained directly from food [1]. 
To maintain oil quality after processing, the fresh bottle samples require correct storage conditions. Oil oxidation is problematic, reducing the oil quality over the storage period due to the breakdown of essential fatty acids, resulting in increased free fatty acids, resulting in oil rancidity, reducing the nutritional benefits significantly [10]. Gómez-Alonso et al. (2007) and Kiralan and Ramadan state that oil quality maintains better in dark and cool conditions for 12 months than storage at ambient temperature. Wroniak and Rękas reported similar results concerning peroxide value and total phenolic content but reported no changes in the fatty acid composition of closed rapeseed oils stored at $4^{\circ} \mathrm{C}$ for 12 months storage [5]. Moreover, it was noted that packing material does not affect the quality of the oil in terms of fatty acid composition or oxidative stability but the availability of oxygen to mix and increase free space within the oil deteriorates the oil during use and storage periods $[4,5]$.

Ireland imports many culinary oils annually; in 2017, Ireland imported 131,000 tons of olive oil. However, Zahoor and Forristal suggest that Ireland could produce more of its culinary oil due to its agricultural background and ideal climatic to cultivate rapeseed as part of annual crop production [11]. This can be financially beneficial to the economy and agricultural sector while also making nutritional and healthy benefiting culinary oils for consumers. The quality of an oil is directly influenced by the cultivar, cultivation, harvesting, and processing techniques. An oil of high quality contains nutritional benefits such as a high USFA content and high antioxidant capacity $[12,13]$. For many years, culinary oils have been studied to profile the nutritional attributes oils contribute to consumers. The fatty acid profile of oil is directly related to the nutritional quality an oil possesses. Many studies have comprehensively characterised the nutritional potential of rapeseed oil based on essential fatty acid composition [14-17]. If the Irish rapeseed oils being studied here demonstrate abundance in essential fatty acid content, the nutritional quality of rapeseed oil from Ireland can be considered a significant factor in producing Irish rapeseed oils.

Routinely fatty acid determination has been carried out using gas chromatography mass spectroscopy (GCMS) techniques. However, these methods require the derivisation and alteration of samples. Bromke et al. found LCMS detection of fatty acids comparable to GCMS, where samples only needed to be filtered before injection. Besides, LCMS allowed for the differentiation between isomers and the separation of fatty acids as large as 26 carbons in length. It was noted that while the precision and correlation with standard peaks tend to be higher for GCMS, peak detection and annotation does not require standards for LCMS detection. Several studies have implemented LCMS for fatty acid detection [18].

While many studies have been conducted characterising culinary rapeseed oils' fatty acid composition, no comprehensive research has been undertaken on those produced in Ireland. The composition of the fatty acids in the oil can directly impact consumers' health [14]. Therefore, the fatty acid composition is a valuable guide to highlight the potential benefits of culinary oil for consumer consumption. Particularly in SFA and total USFA and PUFA content, highlighting the least and most desirable components of an oil's fatty acid composition from a consumer's perspective.

This study aims to conduct a fatty acid analysis of Irish rapeseed oils from 6 Irish producers, focused on the total fatty acid content and the individual fatty acid profile from each rapeseed oil sample. This will determine whether significant differences between culinary rapeseed oils from different Irish producers exist. Additionally, differences within successive batches from each producer may indicate variance with the consistency of the fatty acid content of the rapeseed oils produced from period to period.

\section{Materials and Methods}

2.1. Samples. Commercially available samples of coldpressed Irish rapeseed oils were selected from six Irish rapeseed producers available in local supermarkets and were purchased for the study. Samples were randomly assigned a number 1 to 6 with the specific code PRO1-PRO6, while each "batch" of the samples was coded B1-B3. Individual batches were determined by different processing dates on each bottle and ranked based on the processing date, i.e., batch 1 had the earliest processing date printed.

The coding system was as follows: PRO1B1 represents the rapeseed oil batch produced at the earliest point of the 3 samples by producer 1. Each "batch" was evaluated in triplicate where $n=18$ samples in total.

2.2. Sample Preparation and LCMS Conditions. Fatty acid profiling was carried out based on the method outlined by Otero et al. with slight modifications [19]. This modification included pure samples rather than extracted lipids as the oils are primarily composed of lipids. Each oil sample was diluted 20 times in a mixture of methanol and dichloromethane $(2: 1)$, and $10 \mu \mathrm{L}$ of the diluted sample was injected into the HPLC system equipped with a Q-TOF mass spectrometer (Agilent 6520 series), in triplicate for analysis [19]. Samples were resolved by an Agilent C-18 Poroshell 120 column $(2.7 \mu \mathrm{m}, 3.0 \times 150 \mathrm{~mm})$ separation analytical column. The mobile phase used was a gradient elution, and mobile phase $\mathrm{A}$ consisted of ultrapure water and $2 \mathrm{mM}$ ammonium acetate. Mobile phase B consisted of a $2 \mathrm{mM}$ ammonium acetate mixture in 95\% acetonitrile. The MS was operated in a negative ionisation mode, scanning from 50 to $1,100 \mathrm{~m} / z$. The fragmentor voltages were kept at 175 and $65 \mathrm{~V}$, respectively. Drying gas flow rate, temperature, and nebuliser pressure were at $5 \mathrm{~L} \mathrm{~min}^{-1}, 325^{\circ} \mathrm{C}$, and $0.21 \mathrm{MPa}$, respectively. Peak evaluation and sample screening were conducted using Agilent Mass Hunter Qualitative analysis Navigator software version B.08.00. Standard fatty acids such as palmitic acid (C16:0) and oleic acid (C18:1n9 cis) were used to validate the LC-MS method for fatty acids analysis by comparing their retention time (RT) and specific, accurate mass (Sigma, Ireland). All fatty acids in the samples were then identified based on their known accurate mass, and their relative content was recorded as per their peak area. The peak area of each fatty acid was the average of triplicate samples. 


\section{Results and Discussion}

3.1. Data Analysis. Samples of cold-pressed rapeseed oil from Irish producers were analysed for fatty acid composition. Each sample was injected in triplicate, and the mean peak area for each fatty acid was used to generate the peak percentage for each fatty acid. Known standards were used as guidelines for retention time and $\mathrm{m} / z$ comparison for the fatty acid in the oil samples. Figure 1 illustrates the oleic acid (C18:1) standard used at $10 \mu \mathrm{g} / \mathrm{mL}$ concentration. The chromatogram contains a single peak at approximately 8.47 minutes. The chromatogram's inset depicts the parent ion for oleic acid (C18:1) with an $\mathrm{m} / z$ value of 281.249 in negative ionisation mode, which matched the library as the $\mathrm{m} / z$ of oleic acid $(\mathrm{C} 18: 1)$ is 282.249 .

Likewise, the $\mathrm{m} / z$ of peaks detected in samples were compared to a library of 38 known fatty acids ranging from 8 to 24 carbons chains and varying in saturation. Table 1 outlines the theoretical $\mathrm{m} / z$ to the experimental $\mathrm{m} / z$ detected of the individual fatty acids [19].

A positive match to the library was a minimum match of three significant figures to parent ions of the library values. Similar retention time to the standards was also considered with allowances for matrix interference. Each sample was analysed in triplicate, and mean values were used to calculate the percentage of each fatty acid present within the identified profile of fatty acids (a combination of all fatty acids detected $=100 \%$ ). Figure 2 outlines the separation of fatty acids in a representative sample PRO1B1, where the abundant fatty acids are highlighted in the inset of the chromatogram.

Parent ions and retention times of the fatty acids in the rapeseed oil samples were comparable to the oleic acid (C18:1) standard in Figure 1.

3.2. Fatty Acid Composition of Irish Rapeseed Oils. Fatty acid profiling was executed in triplicate for three batches of rapeseed oil per Irish producer $(n=54)$. Table 2 outlines the dominant compositional fatty acids for each rapeseed oil tested. The total SFAs and USFAs were calculated for each batch.

Fatty acids were detected and reported in terms of the percentage of fatty acid per sample. When combined, each fatty acid percentage yields the total identifiable fatty acid content $(100 \%)$. The fatty acid composition of Irish rapeseed oils tested comprised a mixture of saturated and unsaturated fatty acids. Palmitic acid (C16:0), stearic acid (C18:0), oleic acid (C18:1), linoleic acid (C18:2), linolenic acid (C18:3), and gadoleic acid (C20:0) were the most predominant fatty acids composing each rapeseed oil profile. Traces of other fatty acids make up a smaller proportion of each oil. The trace fatty acids range from $1.40 \%$ to $5.30 \%$, indicating the primary compositional fatty acids construct $94.70 \%$ to $98.60 \%$ of the total profile.

3.3. Saturated Fatty Acids. The SFA content of the Irish rapeseed oils ranged from $6.1 \%$ to $15.8 \%$, where the rapeseed oil from producer 2, B2, had the lowest SFA content, and the rapeseed oil from producer $4, \mathrm{~B} 1$, had a much higher SFA composition (Table 2). Many studies have reported the SFA (\%) content of rapeseed oils ranging from $6.14 \%$ to $9.48 \%[7,14,20,21]$. Five of the 6 Irish rapeseed oils tested had a total SFA content agreeing with these reported values. In contrast, the rapeseed oil from producer $4, \mathrm{~B} 1$, contained a significantly higher SFA content $(p<0.05)$.

Palmitic acid (C16:0) and stearic acid (C18:0) are the most abundant SFA in the rapeseed oils tested. Palmitic acid (C16:0) content is more significant than stearic acid (C18:0), which corresponds with the data reported by Frančáková et al. and Potočnik et al. The palmitic acid (C16:0) content of rapeseed oils tested ranged from $2.21 \%$ to $7.99 \%[7,22$, 23 . The rapeseed oil from producer $2, \mathrm{~B} 2$, had the lowest palmitic acid (C16:0) content, and the rapeseed oil from producer $4, \mathrm{~B} 1$, had the highest. ANOVA analysis determines the difference in palmitic acid (C16:0) be considered statistically significant $(p \leq 0.05)$. Stearic acid $(\mathrm{C} 18: 0)$ content ranged from $1.48 \%$ to $4.34 \%$. The rapeseed oil from producer $6, \mathrm{~B} 2$, had the highest content, and the rapeseed oil produced by producer $3, \mathrm{~B} 1$, had the lowest content of stearic acid (C18:0). These differences, too, was deemed statistically significant (where $p \leq 0.05$ ). These findings agree with data from various literature that reported palmitic acid (C16:0) content in rapeseed oil varying from $4.06 \%$ to $5.20 \%$, and stearic acid (C18:0) was ranging from $1.31 \%$ to $4.40 \%[7,14,22,24]$.

Increased SFA similar to those observed with PRO4B1 can arise at various production stages. Szczepanek et al. and Onemli stated that the organic matter in the soil might influence the fatty acids in the final oil content, specifically, individual fatty acids such as palmitic acid (C16:0) [25, 26]. Joughi et al. determined that late sowing of seeds can increase the SFA proportion of the oil content by increasing palmitic acid (C16:0) and stearic acid (C18:0) [27]. Moisture content influences free fatty acid production, but humidity during storage can also lead to further lipase production, increase the free fatty acids, and reduce the oil's stability. Additionally, environmental factors during cultivation, such as heat and light intensity, are essential in fatty acid development, mainly oleic acid (C18:1) and palmitic acid (C16:0) $[28,29]$. Any of these factors may explain the variations in palmitic and stearic acid (C18:0) concentrations observed in this study. Furthermore, it can be assumed the rapeseed crop from which these oils were produced was cultivated in different geographical regions in Ireland, which can vary in soil type, weathering, and altitude. These differences may affect the development of rapeseed crops and oil fatty acid content and quality.

SFA are undesirable fatty acids associated with increased cholesterol in the blood. Literature has found that a specific cholesterol ratio to HDL in the bloodstream is a more precise heart disease indicator than just LDL concentration [30]. This study also stated that individual fatty acids could affect the ratio. Fatty acids such as myristic and lauric can cause plasma increases, whereas palmitic and stearic acid (C18:0) only slightly influence the ratio. Thus, if the SFA content of rapeseed oil is composed primarily of palmitic acid (C16:0) and stearic acid (C18:0), it can be assumed the effect of rapeseed oil on the cholesterol to HDL ratio 


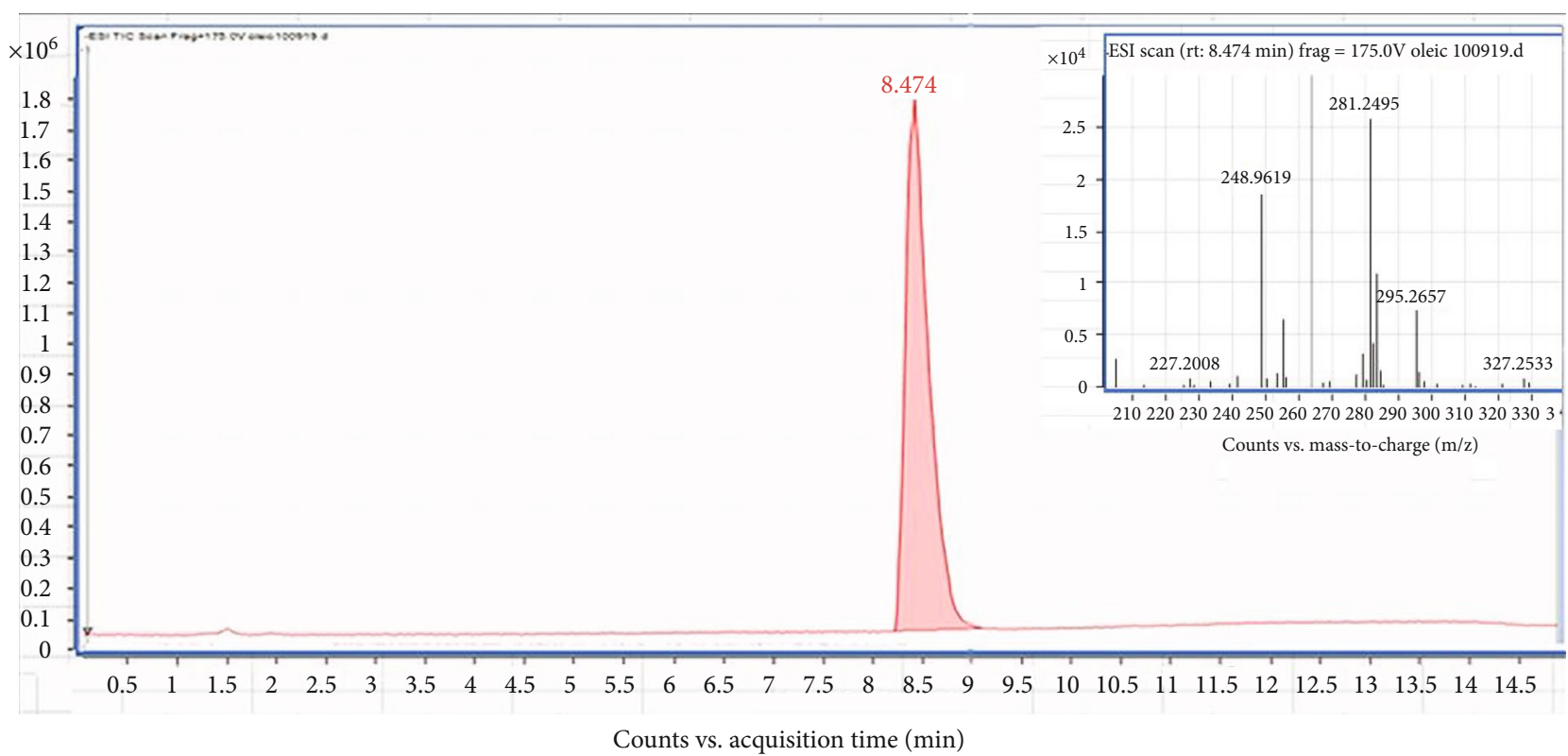

FIGURE 1: Representative ESI TIC peak and corresponding mass spectra (inset image) of standard oleic acid (C18:1) showing characteristic $\mathrm{m} / z$ value (281.249) and retention time $(8.47 \mathrm{~min})$.

would be minimal, if at all. Additionally, Behenic acid is presented in a minor amount in rapeseed oil $(0.37 \%)$ and has been detected in low quantities in some Irish rapeseed oils. Konuskan et al. state that due to the little bioavailability of behenic acid due to its long-chain structure, it would cause less effect on cholesterol content [6].

3.4. Unsaturated Fatty Acids. The USFA content of rapeseed oils accounts for approximately $90 \%$ of the fatty acid composition $[22,30]$. This was evident for rapeseed oils tested in this study. The USFA content ranged from $84.2 \%$ to $93.9 \%$, where significant variations were observed in the USFA subgroups MUFA and PUFA, as outlined in Table 2.

3.4.1. Monounsaturated Fatty Acids (MUFA). Due to a desirable USFA content, rapeseed oil is one of the most consumed oils due to this culinary oil's dominant MUFA content [15]. Of the rapeseed oils tested, the MUFA content consisted primarily of oleic acid (C18:1), linoleic acid (C18:2), linolenic acid (C18:3) and gadoleic acid (C20:0). Oleic acid (C18:1) is the most dominant fatty acid, corresponding with the data reported from the various studies $[14,15,31]$. MUFA content of Irish rapeseed oils varied from $40.95 \%$ to $82.38 \%$, with oleic acid (C18:1) content ranging from $34.17 \%$ to $66.03 \%$ (Table 1), corresponding with the data reported in various studies [32-34]. The oleic acid (C18:1) content variation was considered a statistically significant difference $(p=0.05)$. The rapeseed oil from producer 4, B1, had a lower oleic acid (C18:1) content than the other Irish rapeseed oils tested. It contains lower oleic acid (C18:1) content (45.16\%) and has higher SFA $(11.27 \%)$, which is comparable with data reported on rapeseed oils' oxidation process [35]. Todorov reported variance in the fatty acid content of the same variant of rapeseed crops treated with different fertilisers during cultivation. It was reported that the fatty acid composition, specifically the USFA content, can be directly influenced by cultivation practices resulting in increased USFA composition. Todorov reported USFA content of the same variant of rapeseed ranging from $93.77 \%$ to $95.25 \%$ [7]. This may be an insight into why the MUFA content of the Irish rapeseed oils differ.

3.4.2. Polyunsaturated Fatty Acids (PUFA). PUFA content ranged from $9.14 \%$ to $50.69 \%$ in the Irish rapeseed oil samples tested, which was considered a statistically significant difference $(p=0.001)$. Linoleic acid (C18:2) and $\alpha$-linolenic acid (C18:3) were the main compositional PUFAs detected, ranging from $13.00 \%$ to $40.99 \%$ and $7.85 \%$ to $14.78 \%$, respectively, as per Table 2 . The varying linoleic acid (C18:2) and linolenic acid (C18:3) content compared to data reported by other studies where the linoleic acid and linolenic acid content reported in the studies ranged from $20.24 \%$ to $2.65 \%$ and $8.71 \%$ to $9.56 \%$, respectively [22, 24,35$]$. Some of the Irish rapeseed oils contained considerably higher linoleic acid and linolenic acid content than the rapeseed oils of Greek, Slovakian and Turkish origin. The variation in linoleic acid (C18:2) content was considered statistically significant, while the differences in linolenic acid (C18:3) content were not considered statistically different $(p<0.05)$. Hernández et al. reported that fatty acid desaturase could be responsible for variations in fatty acid composition. Hernández et al. suggested that when environmental temperatures are lower than average, the PUFA content can increase in plants and, therefore, in the seeds' oils. Thus, this could be why the linoleic acid (C18:2) content of Irish rapeseed oils was higher than the earlier report [36].

PUFAs are essential fatty acids to have in culinary oils as the health impact of the oil increases with these essential fatty acids. Linoleic acid (C18:2) is a precursor for arachidic acid, a precursor for eicosapentaenoic acid (EPA). While 
TABle 1: Database of fatty acid information used to analyse chromatograms. The theoretical $\mathrm{m} / z$ of individual fatty acids and the experimental $\mathrm{m} / z$ detected of the individual fatty acids.

\begin{tabular}{|c|c|c|c|c|c|}
\hline Fatty acid & Molecular formula & $\mathrm{C}: \mathrm{U}$ & Molecular mass (g/Mol) & $\mathrm{m} / z(\mathrm{M}-\mathrm{H})^{-}$theoretical & $\mathrm{m} / z(\mathrm{M}-\mathrm{H})^{-}$experimental \\
\hline Caprylic & C8 H16 O2 & $08: 00$ & 144.12 & 143.108 & 143.108 \\
\hline Pelargonic & C9 H18 O2 & $09: 00$ & 158.13 & 157.123 & 157.123 \\
\hline Capric & $\mathrm{C} 10 \mathrm{H} 20 \mathrm{O} 2$ & $10: 00$ & 172.15 & 171.139 & n.d \\
\hline Undecylic & $\mathrm{C} 11 \mathrm{H} 22 \mathrm{O} 2$ & $11: 00$ & 186.16 & 185.155 & n.d \\
\hline Lauric & $\mathrm{C} 12 \mathrm{H} 24 \mathrm{O} 2$ & $12: 00$ & 200.18 & 199.17 & 199.170 \\
\hline Tridecylic & $\mathrm{C} 13 \mathrm{H} 26 \mathrm{O} 2$ & $13: 00$ & 214.19 & 213.186 & n.d \\
\hline Myristic & $\mathrm{C} 14 \mathrm{H} 28 \mathrm{O} 2$ & $14: 00$ & 228.21 & 227.202 & 227.202 \\
\hline Pentadecylic & $\mathrm{C} 15 \mathrm{H} 30 \mathrm{O} 2$ & $15: 00$ & 242.22 & 241.217 & 241.217 \\
\hline Palmitic & $\mathrm{C} 16 \mathrm{H} 32 \mathrm{O} 2$ & $16: 00$ & 256.24 & 255.233 & 255.233 \\
\hline Palmitoleic & $\mathrm{C} 16 \mathrm{H} 30 \mathrm{O} 2$ & $16: 01$ & 254.22 & 253.217 & 253.217 \\
\hline Palmitelaidic & $\mathrm{C} 16 \mathrm{H} 30 \mathrm{O} 2$ & $16: 01$ & 254.22 & 253.217 & n.d \\
\hline Margaric & $\mathrm{C} 17 \mathrm{H} 34 \mathrm{O} 2$ & $17: 00$ & 270.26 & 269.249 & 269.249 \\
\hline Stearic & $\mathrm{C} 18 \mathrm{H} 36 \mathrm{O} 2$ & $18: 00$ & 284.27 & 283.264 & 283.264 \\
\hline Oleic & $\mathrm{C} 18 \mathrm{H} 34 \mathrm{O} 2$ & $18: 01$ & 282.26 & 281.249 & 281.249 \\
\hline Elaidic & $\mathrm{C} 18 \mathrm{H} 34 \mathrm{O} 2$ & $18: 01$ & 282.26 & 281.249 & n.d \\
\hline Linoleic & $\mathrm{C} 18 \mathrm{H} 32 \mathrm{O} 2$ & $18: 02$ & 280.24 & 279.233 & 279.233 \\
\hline Linolenic $(\alpha)$ & $\mathrm{C} 18 \mathrm{H} 30 \mathrm{O} 2$ & $18: 03$ & 278.22 & 277.217 & 277.217 \\
\hline Stearidonic & $\mathrm{C} 18 \mathrm{H} 28 \mathrm{O} 2$ & $18: 04$ & 276.21 & 275.202 & 275.202 \\
\hline Nonadecylic & C19 H38 O2 & $19: 00$ & 298.29 & 297.28 & n.d \\
\hline Arachidic & $\mathrm{C} 20 \mathrm{H} 40 \mathrm{O} 2$ & $20: 00$ & 312.3 & 311.296 & 311.296 \\
\hline Gadoleic & C20 H38 O2 & $20: 01$ & 310.29 & 309.28 & \multirow{2}{*}{309.287} \\
\hline Gondoic & $\mathrm{C} 20 \mathrm{H} 38 \mathrm{O} 2$ & $20: 01$ & 310.29 & 309.28 & \\
\hline Dihomolinoleic & $\mathrm{C} 20 \mathrm{H} 36 \mathrm{O} 2$ & $20: 02$ & 308.27 & 307.264 & 307.264 \\
\hline Dihomolinolenic & C20 H34 O2 & $20: 03$ & 306.26 & 305.249 & 305.249 \\
\hline Mead acid & $\mathrm{C} 20 \mathrm{H} 34 \mathrm{O} 2$ & $20: 03$ & 306.26 & 305.249 & n.d \\
\hline Arachidonic & C20 H32 O2 & $20: 04$ & 304.24 & 303.233 & 303.233 \\
\hline Eicosatetraenoic & $\mathrm{C} 20 \mathrm{H} 32 \mathrm{O} 2$ & $20: 04$ & 304.24 & 303.233 & n.d \\
\hline EPA & $\mathrm{C} 20 \mathrm{H} 30 \mathrm{O} 2$ & $20: 05$ & 302.25 & 301.217 & n.d \\
\hline Heneicosylic & $\mathrm{C} 21 \mathrm{H} 42 \mathrm{O} 2$ & $21: 00$ & 326.32 & 325.311 & n.d \\
\hline Behenic & $\mathrm{C} 22 \mathrm{H} 44 \mathrm{O} 2$ & $22: 00$ & 340.33 & 339.327 & 339.320 \\
\hline Eruic & $\mathrm{C} 22 \mathrm{H} 42 \mathrm{O} 2$ & $22: 01$ & 338.32 & 337.311 & 337.311 \\
\hline Docosadienoic & $\mathrm{C} 22 \mathrm{H} 40 \mathrm{O} 2$ & $22: 02$ & 336.3 & 335.296 & 335.296 \\
\hline Eranthic & $\mathrm{C} 22 \mathrm{H} 38 \mathrm{O} 2$ & $22: 03$ & 334.29 & 333.28 & n.d \\
\hline Ardenic & $\mathrm{C} 22 \mathrm{H} 36 \mathrm{O} 2$ & $22: 04$ & 332.27 & 331.264 & n.d \\
\hline DPA & $\mathrm{C} 22 \mathrm{H} 34 \mathrm{O} 2$ & $22: 05$ & 330.26 & 329.249 & n.d \\
\hline DHA & $\mathrm{C} 22 \mathrm{H} 32 \mathrm{O} 2$ & $22: 06$ & 328.24 & 327.233 & n.d \\
\hline Tricosylic & $\mathrm{C} 23 \mathrm{H} 46 \mathrm{O} 2$ & $23: 00$ & 354.35 & 353.343 & n.d \\
\hline Lignoceric & C24 H48 O2 & $24: 00$ & 368.37 & 367.358 & 367.358 \\
\hline
\end{tabular}

linolenic acid (C18:3) is a precursor for EPA and docosahexaenoic acid (DHA). EPA and DHA are long-chain PUFAs, synthesised from $\alpha$-linolenic acid (C18:3), associated with lowering cardiovascular illnesses, arthritis and inflammatory diseases[15]. Thus, the increased content of PUFA in the Irish rapeseed oil provides the desired health characteristic associated with consuming these culinary oils.

3.5. Variance in Batch Fatty Acids. Differences between the producer batches were evident in this study also. Oleic acid
(C18:1) and linoleic acid (C18:2) demonstrated significant differences within the successive batches. Batch 3 samples demonstrated less oleic acid (C18:1) and more or equal linoleic acid (C18:2) than batches $1 \& 2$. ANOVA statistical analysis proved this a significant difference (where $p \leq 0.05$ ). Additionally, stearic acid (C18:0) content proved to be statistically different within successive batches also. The differences observed in Table 2 for palmitic acid (C16:0), linolenic acid (C18:3), gadoleic acid (C20:0), and trace fatty acids within successive batches were not considered to be 


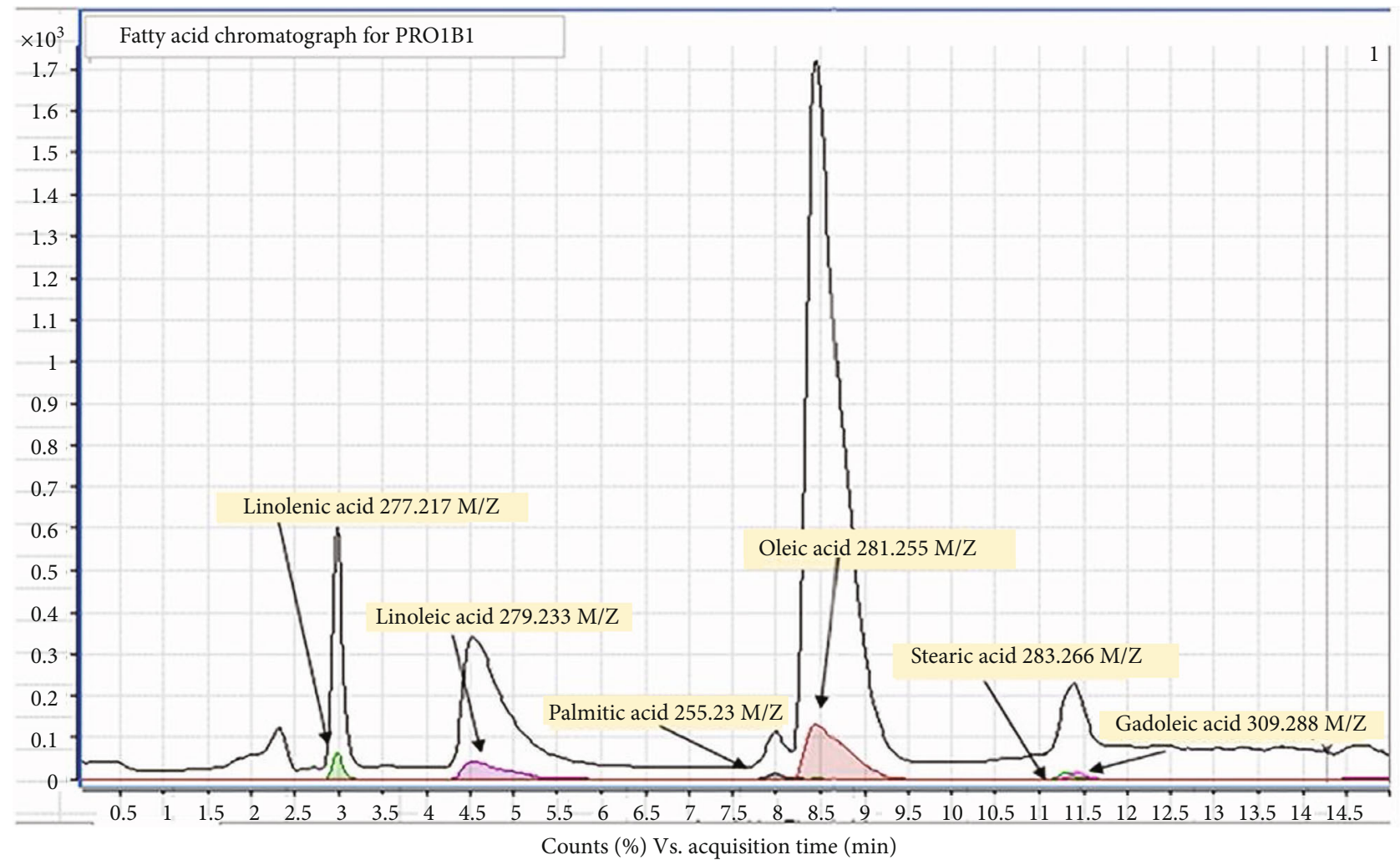

FIGURE 2: Green: linolenic acid (C18:3); purple: linoleic acid (C18:2); grey: palmitic acid (C16:0); red: oleic acid (C18:1); lime: stearic acid (C18:0); magenta: gadoleic acid (C20:0). Separating individual fatty acids in the rapeseed oil from producer 1, batch 1, via LCMS as outlined in Materials and Methods. Coloured peaks represent overlayed chromatograms of identified individual fatty acids separated and detected in PRO1B1.

TABLE 2: Fatty acid profile of rapeseed oil batches $(n=3)$ outlining the primary compositional fatty acids.

\begin{tabular}{|c|c|c|c|c|c|c|c|c|c|c|}
\hline \multicolumn{5}{|c|}{ Saturated fatty acids (\%) } & \multicolumn{6}{|c|}{ Unsaturated fatty acids (\%) } \\
\hline Producers & Palmitic & Stearic & Other & Total SFA & Oleic & Linoleic & Linolenic $(\alpha)$ & Gadoleic & Other & Total USFA \\
\hline PRO1B1 & 3.12 & 2.60 & 1.91 & 7.6 & 58.66 & 22.43 & 8.26 & 2.85 & 0.19 & 92.4 \\
\hline PRO1B2 & 2.67 & 3.47 & 0.73 & 6.9 & 59.75 & 16.58 & 14.50 & 2.03 & 0.26 & 93.1 \\
\hline PRO1B3 & 4.41 & 3.34 & 1.44 & 9.2 & $34.17^{*}$ & $38.44^{*}$ & 14.13 & 3.46 & 0.62 & 90.8 \\
\hline PRO2B1 & 4.01 & 1.97 & 0.70 & 6.7 & 54.86 & 20.86 & 11.05 & 3.66 & 2.88 & 93.3 \\
\hline $\mathrm{PRO} 2 \mathrm{~B} 2$ & 2.21 & 2.67 & 1.26 & 6.1 & 62.05 & 13.17 & 9.59 & 4.74 & 4.32 & 93.9 \\
\hline $\mathrm{PRO} 2 \mathrm{~B} 3$ & 2.53 & 2.33 & 1.99 & 6.8 & $38.10^{*}$ & $32.89^{*}$ & 14.31 & 3.09 & 4.75 & 93.2 \\
\hline PRO3B1 & 5.75 & 1.48 & 0.99 & 8.2 & 57.73 & 20.71 & 10.90 & 2.16 & 0.28 & 91.8 \\
\hline PRO3B2 & 2.76 & 2.72 & 0.82 & 6.3 & 61.95 & 13.00 & 14.78 & 3.75 & 0.23 & 93.7 \\
\hline PRO3B3 & 2.96 & 2.97 & 1.94 & 7.9 & $38.30^{*}$ & $38.50^{*}$ & 13.54 & 1.47 & 0.33 & 92.1 \\
\hline PRO4B1 & 7.99 & 3.93 & 3.85 & $15.8 *$ & 43.77 & 26.93 & 8.17 & 4.35 & 1.01 & $84.2^{*}$ \\
\hline PRO4B2 & 3.89 & 3.49 & 1.80 & 9.2 & 56.25 & 17.60 & 12.70 & 3.89 & 0.36 & 90.8 \\
\hline PRO4B3 & 3.67 & 2.65 & 2.55 & 8.9 & $35.47^{*}$ & $38.99^{*}$ & 13.43 & 2.89 & 0.36 & 91.1 \\
\hline PRO5B1 & 4.43 & 1.54 & 0.84 & 6.8 & 66.03 & 15.89 & 9.99 & 1.05 & 0.22 & 93.2 \\
\hline PRO5B2 & 3.62 & 4.03 & 1.97 & 9.6 & 58.23 & 15.39 & 13.95 & 2.47 & 0.35 & 90.4 \\
\hline PRO5B3 & 3.51 & 3.47 & 2.34 & 9.3 & $41.41^{*}$ & $37.89^{*}$ & 9.97 & 1.11 & 0.30 & 90.7 \\
\hline PRO6B1 & 4.24 & 1.76 & 1.16 & 7.2 & 64.39 & 17.88 & 9.26 & 1.13 & 0.19 & 92.8 \\
\hline PRO6B2 & 4.49 & 4.34 & 1.07 & 9.9 & 45.73 & 33.35 & 7.85 & 3.12 & 0.04 & 90.1 \\
\hline PRO6B3 & 3.69 & 2.30 & 1.40 & 7.4 & $40.60^{*}$ & $40.99^{*}$ & 10.16 & 0.53 & 0.33 & 92.6 \\
\hline
\end{tabular}

$*$ Significantly different $(p<0.05)$. 


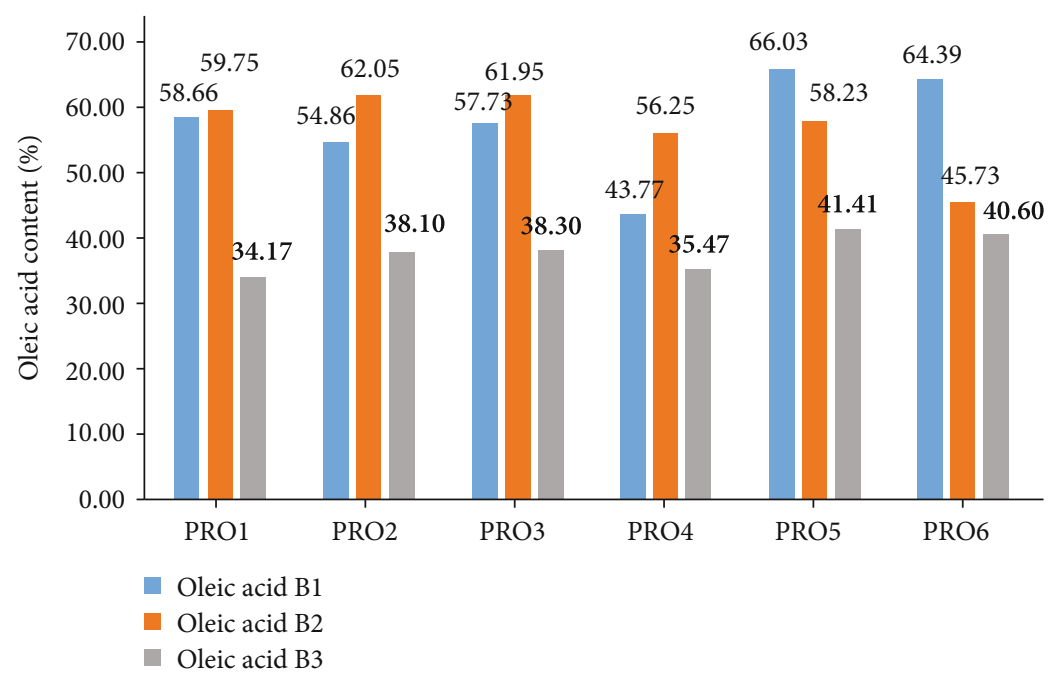

(a)

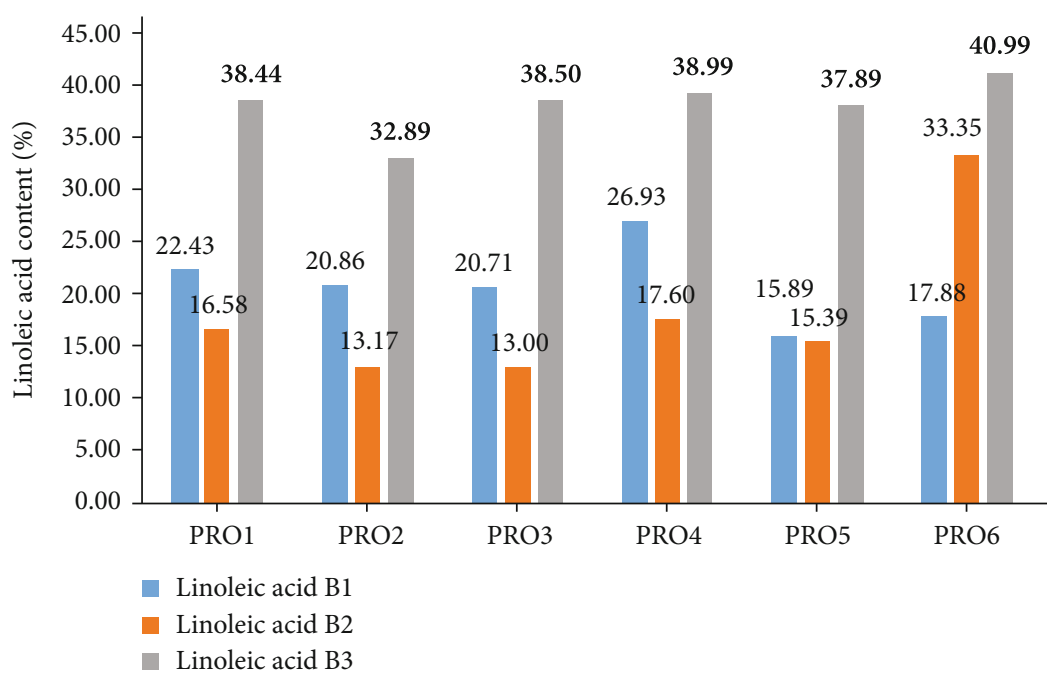

(b)

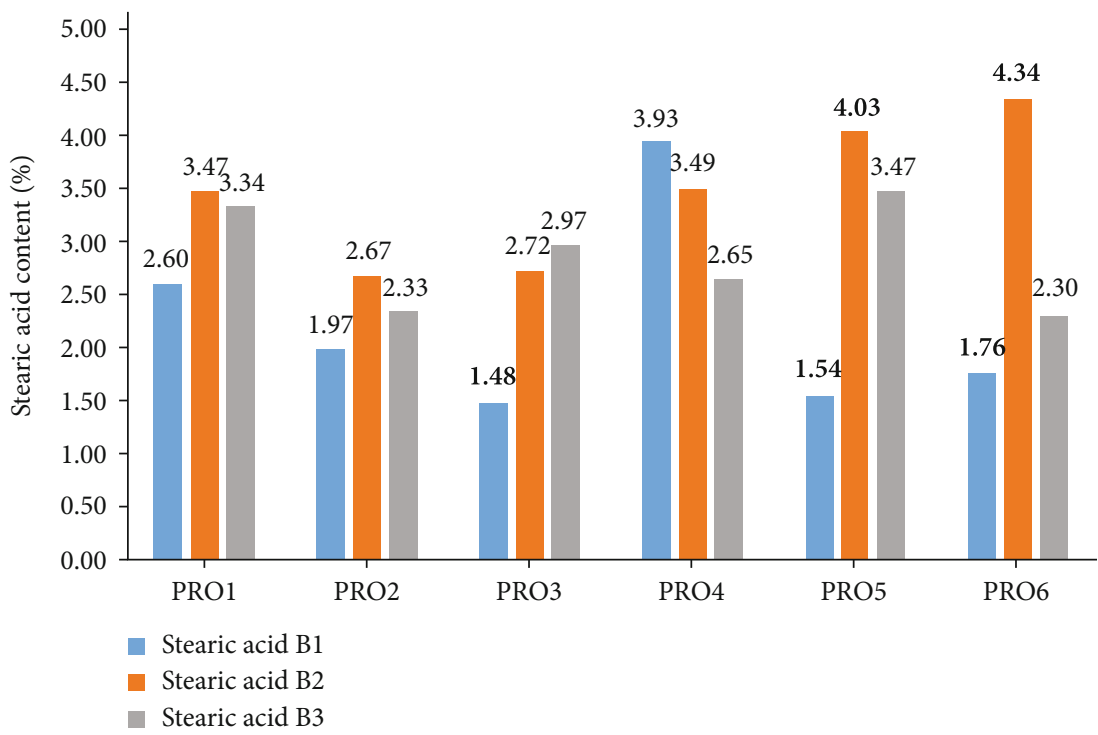

(c)

Figure 3: The statistical differences observed for oleic acid (C18:1), linoleic acid (C18:2), and stearic acid (C18:0) within successive producer batches ( $p \leq 0.05$ ): (a) oleic acid variation within batches, (b) linoleic acid variation within batches, and (c) stearic acid variation within batches. 


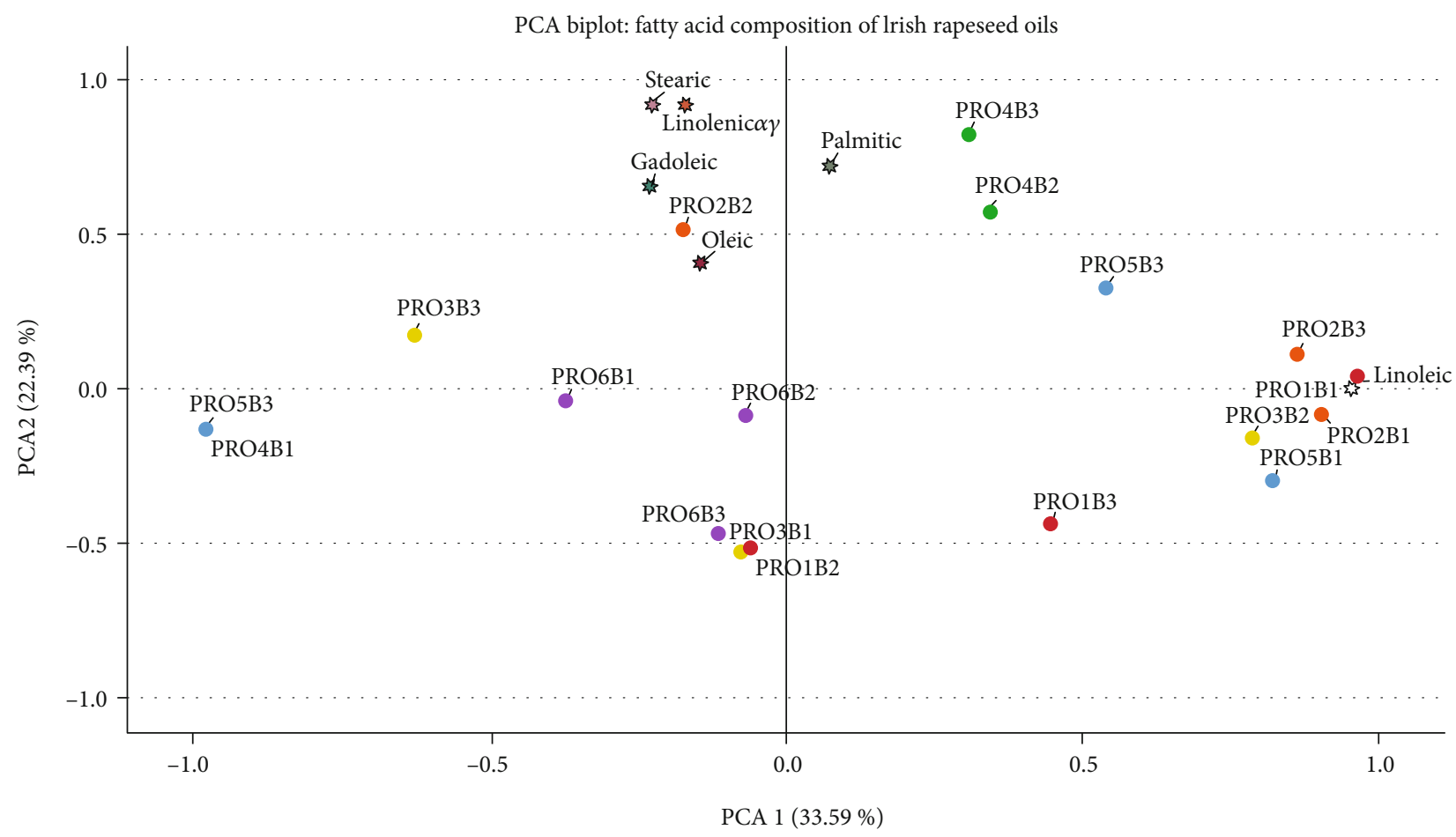

Figure 4: Principle Component Analysis (PCA) biplot of the fatty acid composition of rapeseed oil batches $(n=3)$ with individual fatty acids identified. PCA1 accounted for $33.59 \%$ of the variance observed in the Irish rapeseed oil batches' fatty acid profile, while PCA2 accounted for 22.39\% of these variances. PRO1: red; PRO2: orange; PRO3: yellow; PRO4: green; PRO5: blue; PRO6: purple.

statistically different. A further inspection into the individual fatty acid content may highlight and explain these variations. Figure 3 outlines the statistical differences observed for Oleic acid, linoleic acid and stearic acid within successive batches.

Oleic acid content ranged from $34.17 \%$ to $66.03 \%$, linoleic acid content ranged from $13.00 \%$ to $40.99 \%$, and stearic acid content ranged from $1.48 \%$ to $4.34 \%$ for all samples tested. Oleic acid is more heat-stable than other MUFA, making a high oleic acid containing culinary oils desirable in terms of nutrition and stability during storage and cooking [4]. As observed in Figure 3(a), the third successive batch from each producer exhibits significantly lower oleic acid content (\%). While in Figure 3(b), the third batch demonstrates a significantly higher linoleic acid content (\%). It should be noted that the lower oleic acid content and higher linoleic acid content balance the overall USFA content of the rapeseed oil are maintaining a desirable high USFA content. Figure 3(c) depicts the variation of the SFA stearic acid within batches. No trend can be observed within batch lots, but significant differences can be observed. All significant differences in Figure 3 are highlighted in bold font.

3.6. Variation Comparison. Figure 4 depicts the biplot used to illustrate batch variance based on individual fatty acid content. This Principle Component Analysis (PCA) determined which Irish rapeseed oil batches differentiated from each batch. Two principal components were extracted based on data variance ranging from -1 to +1 . Samples were colour coded per producer, where each producer is represented by
3 , one dots for each successive batch $(n=3)$. Rapeseed oils exhibited similar fatty acid trends grouped in the same component matrix as they were correlated. If the fatty acid content of one oil increased or decreased, the content of the other rapeseed oil exhibited similar trends. Regarding the PCA model, variables positioned close to the component origin (0.0) had a weaker impact on the overall model. Meaning they did not exhibit significant differences within the model trend.

PCA1 contained samples differentiated by palmitic acid (C16:0), the rapeseed oils from producer 4 are distinguishable in this component. These batch samples are positioned on opposite ends of the plot origin, which indicates a negative relationship. The rapeseed oil from producer 4 , batch 1 (PRO3B1), had a significantly higher palmitic acid (C16:0) content than batches 2 or 3 ; therefore, it is justifiable for these samples to be on the opposite ends of the PCA 1 axis. Two rapeseed oils from producer 5 , batch 2 and batch 3 (PRO5B2, PRO5B3), exhibited a similar relationship. While batch one from producer 5 lies within PCA2, indicating the fatty acid content of the rapeseed oil from PRO5B1 differed from the other batches from producer 5. Based on the data in Table 2, PRO5B1 contained less SFA content and higher USFA content than batch 2 and batch 3, which may rationalise why this sample lies in PCA2. While the oils from PRO6B1-B3, PRO3B1, and PRO1B2 distribute through PCA1, they did not contribute significantly to the model as they lie close to the origin. The samples PRO6B3, PRO3B1, and PRO1B2 lie close together in PCA1, indicating that 
these rapeseed oils exhibited similar variation patterns in the PCA model and, therefore, similar fatty acid content.

PCA2 distribution demonstrated variations of fatty acid content based on USFA. PCA2 contained samples differentiated by stearic acid (C18:0), oleic acid (C18:1), linolenic acid (C18:3), and gadoleic acid (C20:0) on one end of the axis. In contrast, linoleic acid (C18:2) resided at the opposite end, indicating a correlation between these variables. Linoleic acid (C18:2) was positioned close to +1 on the PCA2 axis, indicating that the fatty acid profile of the rapeseed oils in PCA2 is explained more by differences in linoleic acid (C18:2) content. The rapeseed oil from producer 2, batch 2 (PRO2B2), was positioned closer to oleic acid (C18:1) due to the higher oleic acid (C18:1) content this rapeseed oil contained. In contrast, batch 1 from producer 2 (PRO2B1) had a higher linoleic acid (C18:2) content than PRO2B2, which justifies the position of this sample in the PCA plot; these batches were inversely proportional in the model. Batch 3 from producer 2 (PRO2B3) was also positioned in PCA2; however, this batch aligned closer to the PCA1 axis. While this rapeseed oil exhibited a low content of palmitic acid (C16:0), it also contained higher linoleic acid (C18:2) than both batches. Hence, this sample contributed variance in the model's palmitic acid (C16:0) and linoleic acid (C18:2) content.

The position of PRO3B2 and PRO3B3 in PCA2 demonstrates different linoleic acid (C18:2) and oleic acid (C18:1) content, resulting in these samples being distributed on opposite ends PCA2 axis. PRO3B2 had significantly less oleic acid (C18:1) content than PRO3B3 but presented a considerably higher linoleic acid (C18:2) content, as shown in Figure 4.

Based on the findings in this study outlined in Table 2 and confirmed in Figures 3 and 4, rapeseed oils from Irish producers possess high concentrations of desirable fatty acid such as oleic acid (C18:1), linoleic acid (C18:2) and linolenic acid (C18:3) which confirms that rapeseed oils produced in Ireland have a nutritionally beneficial fatty acid composition which can contribute to consumer health. Additionally, this study highlighted variations in rapeseed oils' fatty acid composition from 6 different Irish producers. Furthermore, these variations can be observed for individual fatty acids of the 3 successive batches per producer.

\section{Conclusion}

The fatty acid content of Irish rapeseed oils was nutritionally beneficial due to the high unsaturated fatty acid proportion of the overall profile and the desirable MUFA and PUFA content these oils possess, which can contribute to consumers health. The fatty acid composition demonstrated significant differences in individual fatty acid content, while total saturation had insignificant differences. The reason for the variation in individual fatty acids of the rapeseed oils tested was unknown. However, as previously mentioned, many studies have contributed cultivation practices, improper processing, harvesting time and geographical regions as influential factors to an oil's fatty acid content.

\section{Abbreviations}

SFA: $\quad$ Saturated fatty acids

USFA: Unsaturated fatty acids

MUFA: Monounsaturated fatty acids

PUFA: Polyunsaturated fatty acids

LCMS: Liquid chromatography-mass spectroscopy

LDL: Low-density lipoprotein

HDL: High-density lipoprotein.

\section{Data Availability}

The data that support the findings of this study are available from the corresponding author upon reasonable request.

\section{Conflicts of Interest}

The authors have no conflict of interest to declare.

\section{Acknowledgments}

The authors would like to thank the Graduate Research Office at the Technical University of the Shannon (TUS) and the Shannon Applied Biotechnology Centre for supporting this research. Additionally, we especially thank Dr Sushanta Saha for his guidance and encouragement to pursue the publication of this work.

\section{References}

[1] T. Jain, "Fatty acid composition of oilseed crops: a review," Emerging Technologies in Food Science, vol. 13, pp. 147-153, 2020.

[2] A. Morales-Martínez, A. Sánchez-Mendoza, J. C. MartínezLazcano et al., "Essential fatty acid-rich diets protect against striatal oxidative damage induced by quinolinic acid in rats," Nutritional Neuroscience, vol. 20, no. 7, pp. 388-395, 2017.

[3] A. C. Tierney and I. Zabetakis, "Changing the Irish dietary guidelines to incorporate the principles of the Mediterranean diet: proposing the MedÉire diet," Public Health Nutrition, vol. 22, no. 2, pp. 375-381, 2019.

[4] S. C. Chew, "Cold-pressed rapeseed (Brassica napus) oil: chemistry and functionality," Food Research International, vol. 131, article 108997, 2020.

[5] M. Wroniak and A. Rękas, "Nutritional value of cold-pressed rapeseed oil during long term storage as influenced by the type of packaging material, exposure to light \& oxygen and storage temperature," Journal of Food Science and Technology, vol. 53, no. 2, pp. 1338-1347, 2016.

[6] D. B. Konuskan, M. Arslan, and A. Oksuz, "Physicochemical properties of cold pressed sunflower, peanut, rapeseed, mustard and olive oils grown in the eastern Mediterranean region," Saudi Journal of Biological Sciences, vol. 26, no. 2, pp. 340-344, 2019.

[7] Z. Todorov, "Composition and Quality of Rapeseed Oil (Brassica napus oleifera biennis) Depending on Sowing Time and Treatment with Leaf Fertilizers," Agronomy Journal, vol. LXIII, no. 1, pp. 574-579, 2020, http://agronomyjournal.usamv.ro/ pdf/2020/issue_1/Art80.pdf.

[8] Z. Mekonnen, A. Gebreselema, and Y. Abere, "Effect of locally manufactured Niger seed oil on lipid profile compared to 
imported palm and sunflower oils on rat models," Journal of Lipids, vol. 2018, no. 1, p. 8, 2018.

[9] S. Namazkar, A. Stockmarr, G. Frenck et al., "Concurrent elevation of $\mathrm{CO} 2, \mathrm{O} 3$ and temperature severely affects oil quality and quantity in rapeseed," Journal of Experimental Botany, vol. 67, no. 14, pp. 4117-4125, 2016.

[10] M. Kiralan and M. F. Ramadan, "Volatile oxidation compounds and stability of safflower, sesame and canola coldpressed oils as affected by thermal and microwave treatments," Journal of Oleo Science, vol. 65, no. 10, pp. 825-833, 2016.

[11] F. Zahoor and D. Forristal, Winter Oilseed Rape, CropQuest, 2015, March 2020, https://www.teagasc.ie/media/website/ crops/crops/OSR-Full-report.pdf.

[12] E. Rokosik, K. Dwiecki, and A. Siger, "The quality of coldpressed rapeseed oil obtained from seeds of Brassica napus L. with increased moisture content," Acta Scientiarum Polonorum. Technologia Alimentaria, vol. 18, no. 2, pp. 205-218, 2019.

[13] E. P. Kalogianni, D. Georgiou, M. Romaidi et al., "Rapid Methods for Frying Oil Quality Determination: Evaluation with Respect to Legislation Criteria," Journal of the American Oil Chemists' Society, vol. 94, no. 1, pp. 19-36, 2017.

[14] J. Orsavova, L. Misurcova, J. V. Ambrozova, R. Vicha, and J. Mlcek, "Fatty acids composition of vegetable oils and its contribution to dietary energy intake and dependence of cardiovascular mortality on dietary intake of fatty acids," International Journal of Molecular Sciences, vol. 16, no. 12, pp. 12871-12890, 2015.

[15] S. Hammad, S. Pu, and P. J. Jones, "Current evidence supporting the link between dietary fatty acids and cardiovascular disease," Lipids, vol. 51, no. 5, pp. 507-517, 2016.

[16] S. Egert, M. Fobker, G. Andersen, V. Somoza, H. F. Erbersdobler, and U. Wahrburg, "Effects of dietary $\alpha$-linolenic acid, eicosapentaenoic acid or docosahexaenoic acid on parameters of glucose metabolism in healthy volunteers," Annals of Nutrition \& Metabolism, vol. 53, no. 3-4, pp. 182-187, 2009.

[17] S. Egert, A. Baxheinrich, Y. H. Lee-Barkey et al., "Effects of a hypoenergetic diet rich in $\alpha$-linolenic acid on fatty acid composition of serum phospholipids in overweight and obese patients with metabolic syndrome," Nutrition, vol. 49, pp. 74-80, 2018.

[18] M. A. Bromke, A. Hochmuth, T. Tohge et al., "Liquid chromatography high-resolution mass spectrometry for fatty acid profiling," The Plant Journal, vol. 81, no. 3, pp. 529-536, 2015.

[19] P. Otero, S. K. Saha, J. M. Gushin, S. Moane, J. Barron, and P. Murray, "Identification of optimum fatty acid extraction methods for two different microalgae Phaeodactylum tricornutum and Haematococcus pluvialis for food and biodiesel applications," Analytical and Bioanalytical Chemistry, vol. 409, no. 19, pp. 4659-4667, 2017.

[20] A. Rękas, M. Wroniak, A. Siger, I. Ścibisz, D. Derewiaka, and A. Anders, "Mechanical hulling and thermal pre-treatment effects on rapeseed oil antioxidant capacity and related lipophilic and hydrophilic bioactive compounds," International Journal of Food Sciences and Nutrition, vol. 68, no. 7, pp. 788-799, 2017.

[21] N. Mikołajczak, M. Tańska, and I. Konopka, "Impact of the addition of 4-vinyl-derivatives of ferulic and sinapic acids on retention of fatty acids and terpenoids in cold-pressed rapeseed and flaxseed oils during the induction period of oxidation," Food chemistry, vol. 278, no. 278, pp. 119-126, 2019.

[22] H. Frančáková, E. Ivanišová, Š. Dráb et al., "Composition of fatty acids in selected vegetable oils," Potravinarstvo, vol. 9, no. 1, pp. 538-542, 2015.
[23] T. Potočnik, N. Ogrinc, D. Potočnik, and I. J. Košir, "Fatty acid composition and $\delta 13 \mathrm{C}$ isotopic ratio characterisation of pumpkin seed oil," Journal of Food Composition and Analysis, vol. 53, pp. 85-90, 2016.

[24] E. G. Giakoumis, “Analysis of 22 vegetable oils' physicochemical properties and fatty acid composition on a statistical basis, and correlation with the degree of unsaturation," Renewable Energy, vol. 126, pp. 403-419, 2018.

[25] M. Szczepanek, E. Wilczewski, and A. Siwik-Ziomek, "Effect of a biostimulant on the accumulation of $\mathrm{mg}$ in winter oilseed rape under different mineral fertilization doses," Journal of Elementology, vol. 22, no. 4, pp. 1375-1385, 2017.

[26] F. Onemli, "Fatty acid content of seed at different development stages in canola on different soil types with low organic matter," Plant Production Science, vol. 17, no. 3, pp. 253-259, 2014.

[27] E. S. G. Joughi, E. M. Hervan, A. H. S. Rad, and G. Noormohamadi, "Fatty acid composition of oilseed rapeseed genotypes as affected by vermicompost application and different thermal regimes," Agronomy Research, vol. 16, no. 1, pp. 230-242, 2018.

[28] S. Dabbou, I. Chaieb, I. Rjiba et al., "Multivariate data analysis of fatty acid content in the classification of olive oils developed through controlled crossbreeding," Journal of the American Oil Chemists' Society, vol. 89, no. 4, pp. 667-674, 2012.

[29] B. D. P. Rondanini, D. N. Castro, P. S. Searles, and M. C. Rousseaux, "Fatty acid profiles of varietal virgin olive oils (Olea europaea L.) from mature orchards in warm arid valleys of northwestern Argentina (La Rioja)," Grasas y Aceites, vol. 62, no. 4, pp. 399-409, 2011.

[30] M. R. Keogh-Brown, H. T. Jensen, S. Basu et al., "Evidence on the magnitude of the economic, health and population effects of palm cooking oil consumption: an integrated modelling approach with Thailand as a case study," Population Health Metrics, vol. 17, no. 1, p. 12, 2019.

[31] D. Lin, M. Xiao, J. Zhao et al., "An overview of plant phenolic compounds and their importance in human nutrition and management of type 2 diabetes," Molecules, vol. 21, no. 10, p. $1374,2016$.

[32] L. Redondo-Cuevas, G. Castellano, F. Torrens, and V. Raikos, "Revealing the relationship between vegetable oil composition and oxidative stability: a multifactorial approach," Journal of Food Composition and Analysis, vol. 66, no. 2, pp. 221-229, 2018.

[33] X. Liu, N. Hoshino, S. Wang, E. Masui, J. Chen, and H. Zhang, "A novel evaluation index for predicting the degradation rate of frying oils based on their fatty acid composition," European Journal of Lipid Science and Technology, vol. 120, no. 7, pp. 16, 2018.

[34] M. Maszewska, A. Florowska, K. Matysiak, K. MarciniakŁUkasiak, and E. Dłuzewska, "The study of palm and rapeseed oil stability during frying," Journal of Applied Botany and Food Quality, vol. 91, no. 1, pp. 103-108, 2018.

[35] E. Beyzi, A. Gunes, S. Buyukkilic Beyzi, and Y. Konca, "Changes in fatty acid and mineral composition of rapeseed (Brassica napus ssp. oleifera L.) oil with seed sizes," Industrial Crops and Products, vol. 129, pp. 10-14, 2019.

[36] M. L. Hernández, M. N. Padilla, M. D. Sicardo, M. Mancha, and J. M. Martínez-Rivas, "Effect of different environmental stresses on the expression of oleate desaturase genes and fatty acid composition in olive fruit," Phytochemistry, vol. 72, no. 2-3, pp. 178-187, 2011. 